\title{
Ein klassisches Modell molekularer Dipole und Polarisierbarkeiten
}

\author{
Von Frank Matossi \\ Naval Ordnance Laboratory, White Oak, Silver Spring, Maryland \\ (Z. Naturforschg. 8a $61-67$ [1953]; eingegangen am 15. September 1952)
}

Herrn Professor Dr. E. Fue sum 60. Geburtstag gewidmet

\begin{abstract}
Molekulare Dipole und Polarisierbarkeiten sind wesentlich bestimmt durch die Mitwirkung und Wechselwirkung induzierter atomarer Dipole. In Erweiterung älterer Modelle werden Abschirmungsfaktoren für die Felder der induzierten Dipole eingeführt. Außerdem werden die atomaren Polarisierbarkeiten als mit dem induzierenden Feld bzw. dem Atomabstand veränderlich angesehen. Auf diese Weise gelingt es, die Beobachtungen über Dipolmomente, Ultrarotintensitäten, Raman- und Rayleigh-Depolarisationsgrade, Molekularrefraktion und Kerr-Effekt mit einem einheitlichen Modell darzustellen. Das Modell wird angewandt zum qualitativen Verständnis der Ultrarotbeobachtungen an homöopolaren Stoffen und an disubstituierten Benzolen.
\end{abstract}

$\mathrm{K}$ lassische Modelle haben auch heute noch einen mindestens heuristischen Wert. Von einem fruchtbaren Modell muß aber verlangt werden, da $\$$ es in sich geschlossen ist und eine ganze Reihe von verwandten Erscheinungen unter einem einheitlichen Gesichtspunkt zu erfassen gestattet. Für das klassische Modell, das die Dipoleigenschaften von Molekülen mit einigen optischen Eigenschaften (Ultrarotabsorption, Rayleigh- und Raman-Streuung, Molekularrefraktion, Kerr-Effekt) zu verbinden suchte, war jedoch diese Forderung nicht erfüllt. Wohl war für jeden einzelnen Effekt ein $\mathrm{Zu}$ sammenhang leicht herzustellen, aber die Modelle waren untereinander nicht verträglich.

Schon das einfache klassische Modell des Dipolmoments eines zweiatomigen Moleküls, $\mu=e r$, versagt quantitativ, wenn $e$ die Elektronenladung und $r$ den Atomabstand darstellen. Man muß eine (,statische") effektive Ladung $\varepsilon=\mu / r$ einführen, die man außerdem von einer ,dynamischen" effektiven Ladung $\varepsilon^{\prime}=\mu^{\prime}$ unterscheiden muß $\left(\mu^{\prime}=\mathrm{d} \mu / \mathrm{d} r\right)$, wenn die aus Ultrarotintensitäten berechenbaren Werte von $\mu^{\prime}$ quantitativ gedeutet werden sollen.

Auch das klassische Silbersteinsche Modell für die molekulare Polarisierbarkeit und deren Anisotropie $^{1}$, die empirisch aus der Molekularrefraktion und dem Depolarisationsgrad der Rayleigh-Streu-

${ }^{1}$ L. Silberstein, Philos. Mag. J. Sci. 33, 92, 215. $521[1917]$.

2 F. Matossi, Physik. Z. 45, 304 [1945]; J. chem. Physics 19, 1007 [1951]: 20, 819 [1952], dort auch weitere Literatur. ung (oder aus dem Kerr-Effekt) gewonnen werden, versagt schon für die eben genannten Erscheinungen, für deren quantitative Interpretation es ja gedacht war, wenn man die heute sehr genau bekannten Werte der Atomabstände einsetzt. Noch weniger ist es in der Lage, die Depolarisationsgrade der Raman-Linien totalsymmetrischer Schwingungen darzustellen, die sich aus der Änderung der molekularen Polarisierbarkeiten ergeben sollten.

In einer Reihe von Arbeiten konnte gezeigt werden, wie man einzelne Schwächen der angedeuteten Modelle beheben kann. Dies geschah im wesentlichen durch die Berücksichtigung des Einflusses und der Wechselwirkung induzierter Dipole und mit der Annahme, daß die atomaren Polarisierbarkeiten vom induzierenden Feld oder, was auf dasselbe hinauskommt, vom Abstand der Atome abhängen sollen ${ }^{2}$. Ferner mußten Abschirmungsfaktoren eingeführt werden ${ }^{3}$, die der Änderung der Felder infolge der Überlappung der Elektronenhüllen Rechnung tragen ${ }^{4}$.

In dem vorliegenden Artikel soll, ohne auf die Einzelheiten einzugehen, das Modell und die Methode seiner Auswertung kurz dargelegt werden. Außerdem soll auf Grund des Modells die Deutung einiger Beobachtungen über Ultrarotintensitäten versucht werden.

${ }^{3}$ F. Matossi, erscheint in J. chem. Physics.

${ }^{4}$ Vgl. N. F. Mott u. R. W. Gurney, Electronic Processes in Ionic Crystals, 2. Aufl., S. 17, Clarendon Press, Oxford 1948. 


\section{Das Modell}

Für das Dipolmoment $\mu$ eines zweiatomigen Moleküls setzen wir

$$
" \prime=\operatorname{er}\left(1-\sigma \frac{\alpha_{1}+\alpha_{2}}{r^{3}}\right),
$$

wo $\alpha_{1}$ und $\alpha_{2}$ die atomaren Polarisierbarkeiten, $r$ der Abstand der Atome und $e$ deren elektrische Ladung sind ${ }^{5}$. $\sigma$ ist ein Abschirmungsfaktor, der von anderer Seite vorgeschlagen wurde ${ }^{6}$.

Gl. (1) beschreibt den Zustand des Dipols als herrührend von der Überlagerung eines Ionendipols und der von den Ionenladungen in den Atomen induzierten Dipole. Tatsächlich existieren keine Ionen im Molekül. Die hier herangezogenen Ionen sind daher fiktiv, und die auftretenden Polarisierbarkeiten sind deshalb auch nicht als mit Ionenpolarisierbarkeiten identisch anzusehen. Der Ansatz soll auch nicht aufgefaßt werden als der Beginn einer Reihenentwicklung nach Potenzen von $\alpha / r^{3}$. Denn das würde bedeuten, daß die induzierten Dipole, die schon das Resultat einerWechselwirkung sind, wiederum aufeinander einwirken, was beliebig fortgesetzt werden könnte. Das wäre nicht recht verständlich und würde außerdem in vielen Fällen zu divergenten Reihen führen.

Die dynamische effektive Ladung ist nun direkt gegeben durch

$$
u^{\prime}=e\left[1+\frac{2 \sigma\left(\alpha_{1}+\alpha_{2}\right)}{r^{3}}-\sigma \frac{\alpha_{1}{ }^{\prime}+\alpha_{2}{ }^{\prime}}{r^{2}}\right] .
$$

wo $\alpha^{\prime}=\mathrm{d} \alpha / \mathrm{d} r$.

Die Einführung von $\alpha^{\prime} \neq 0$ ist der wesentliche Schritt, die effektiven Ladungen numerisch zu beschreiben? ${ }^{7}$ Das Experiment liefert übrigens nur $(\mathrm{d} \mu / \mathrm{d} Q)^{2}$, wo $Q$ die Normalkoordinate der Schwingung ist und nicht $\mu^{\prime}$ selbst. Bei mehratomigen Molekülen, bei denen sich unsere Gleichungen auf Bindungsmomente beziehen, müssen daher die Normalkoordinaten bekannt sein (d.h. die Schwingungsform), um von $\mathrm{d} \mu / \mathrm{d} Q$ auf $\mathrm{d} \mu / \mathrm{d} r$ zu transformieren ${ }^{8}$. Außerdem ist das Vorzeichen von $\mu^{\prime}$ unbestimmt.

Für Atome mit gleichem Vorzeichen der fiktiven Ionenladung (,,homöopolare Moleküle“) kann für

\footnotetext{
5 Ein ähnlicher Ansatz ist schon frühzeitig versucht worden (F. Hund, Z. Physik 31, 81 [1925]; F. M a tossi, Z. Physik 40, 1 [1927], aber die Anwendung litt unter der Unzulänglichkeit der damaligen Daten. Vgl. auch R. Mecke, Z. Elektrochem. 54, 38 [1950].

${ }^{6}$ Ch. K. Møller, J. chem. Physics 20, 203 [1952].
}

$\mu$ und $\mu^{\prime}$ geschrieben werden (Fortfall des Ionenglieds, Umkehrung des einen induzierten Dipols)

$$
\begin{aligned}
& \prime \prime=\sigma \frac{\alpha_{1}-\alpha_{2}}{r^{3}} e, \\
& \mu^{\prime}=\sigma \frac{e}{r^{2}}\left[\alpha_{1}{ }^{\prime}-\alpha_{2}{ }^{\prime}-\frac{2\left(\alpha_{1}-\alpha_{\bullet}\right)}{r}\right] .
\end{aligned}
$$

Die Komponenten $A$ und $B$ der molekularen Polarisierbarkeit eines zweiatomigen Moleküls parallel und senkrecht zur Verbindungslinie der Atome sind gegeben durch

$$
\begin{aligned}
& A=\frac{\alpha_{1}+\alpha_{2}+4 \beta \alpha_{1} \alpha_{2} / r^{3}}{1-4 \beta^{2} \alpha_{1} \alpha_{2} / r^{6}} \\
& B=\frac{\alpha_{1}+\alpha_{2}-2 \gamma \alpha_{1} \alpha_{2} / r^{3}}{1-\gamma^{2} \alpha_{1} \alpha_{2} / r^{6}} .
\end{aligned}
$$

Hier ist die Wechselwirkung der in den beiden Atomen von einem äußeren Feld induzierten Dipole berücksichtigt und wiederum mit Abschirmungsfaktoren $\beta$ oder $\gamma$ versehen worden. Dies ist die wesentliche Änderung gegenüber dem ursprünglichen Silbersteinschen Ansatz, den man für $\beta=$ $\gamma=1$ erhalten würde. Es stellte sich heraus, daß die Einführung der Parameter $\beta$ und $\gamma$ notwendig ist, um die experimentell bestimmten Werte von $A$ und $B$ darzustellen. Um mit demselben Ansatz auch $A^{\prime}=\mathrm{d} A / \mathrm{d} r$ und $B^{\prime}=\mathrm{d} B / \mathrm{d} r$ übereinstimmend mit den Beobachtungen $\mathrm{zu}$ erhalten, muß wiederum $\alpha^{\prime} \neq 0$ angenommen werden.

Für mehratomige Moleküle sind die Ausdrücke (3) komplizierter, und außerdem muß wieder der Einfluß der Schwingungsform in Betracht gezogen werden, indem man z. B. setzt

$$
A^{\prime}=\sum \frac{\partial A}{\partial r_{i k}} \mathrm{~d} r_{i k}, B^{\prime}=\sum \frac{\partial B}{\partial r_{i k}} \mathrm{~d} r_{i k},
$$

wo die Summe über alle Abstände $r_{i k}=r_{k i}$ genommen wird. Die relativen Werte der Abstandsänderungen $\mathrm{d} r_{i k}$ sind durch die Normalkoordinaten der Schwingung gegeben.

Die experimentellen Daten liefern $A, B$ und $A^{\prime} / B^{\prime}$ $A$ und $B$ werden erhalten aus dem Dipolarisationsgrad der Rayleigh-Streuung

$$
\varrho=\frac{6}{7+45(a / b)^{2}} \quad \text { mit } \quad \begin{aligned}
& a=1 / 3(A+2 B), \\
& b=A-B
\end{aligned}
$$

${ }^{7}$ Ein etwas anderes Modell ist von J. Duchesne. Bull. Cl. Sci., Acad. roy. Belgique 38, 197 [1952], vorgeschlagen worden. Er setzt $e$ als Funktion von $r$ an.

${ }^{8}$ Siehe z. B. D. Z. Robinson. J. chem. Physics 19, 881 [1951]. 
und aus der Molekularrefraktion, die proportional zu $a$ ist $^{9}$. Der Depolarisationsgrad der RamanLinien liefert $A^{\prime} \mid B^{\prime}$ aus derselben Gl. (5), in der nur $A$ und $B$ durch $A^{\prime}$ und $B^{\prime}$ ersetzt sind. Absolutwerte von $A^{\prime}$ und $B^{\prime}$ würden durch die Intensitäten der Raman-Linien gegeben sein. Leider entziehen sich die Raman-Intensitäten dem Modell, teils weil überhaupt absolute Intensitätsangaben nicht vorliegen, teils weil möglicherweise die Intensitäten noch durch andere Faktoren als durch $A^{\prime}$ und $B^{\prime}$ allein bedingt sind.

\section{Numerische Auswertung}

Da die Polarisierbarkeiten und die anderen Parameter $(\beta, \gamma, \sigma)$ nicht auf unabhängige Weise bekannt sind, muß die Prüfung und Auswertung des Modells dadurch geschehen, daß diese Parameter auf Grund der experimentellen Daten $\left(\mu, \mu^{\prime}, A, B\right.$, $\left.A^{\prime} \mid B^{\prime}\right)$ berechnet werden. Dies ist nicht ohne Willkür möglich, da meistens mehr unbekannte Parameter vorliegen als experimentelle Bestimmungsstücke. Man wird daher plausible, aber immerhin willkürliche Annahmen machen müssen, wie z. B. Übertragbarkeit der Polarisierbarkeit eines Atoms von einem Molekül auf ein anderes (dies ist keineswegs eine notwendige Folge des Modells) oder $\beta=\gamma$ oder $\gamma=1$. Auch die Wahl des Werts von $e$ ist nicht willkürfrei. Wir haben überall $e=1$ Elementarladung gesetzt, selbst bei Molekülen mit zweiwertigen Atomen, da die üblichen Resonanzstrukturen solcher Moleküle dies nahelegen. Glücklicherweise hat der genaue Zahlenwert von $e$ keinen großen Einfluß auf das Resultat. Møller ${ }^{6}$ benutzt $e=1 / 3$ Elementarladung und setzt für $\alpha$ Ionenpolarisierbarkeiten ein.

Ferner treten Mehrdeutigkeiten wegen der quadratischen Natur mancher Gleichungen auf und wegen der Unbestimmtheit der Vorzeichen von $\mu^{\prime}$ und $a / b$. Wo mehrere Lösungen formal möglich sind, wurde die gewählt, die $\alpha^{\prime}$ einen positiven und nicht zu großen Wert gab. Die obere Grenze von $\alpha^{\prime}$ ist dadurch gegeben, daß die Volumenänderung, die der Änderung von $\alpha$ anschaulich zugeordnet ist, nicht größer werden soll, als der Abstandsänderung infolge der Amplitude der Schwingung entspricht.

${ }^{9} A-B$ kann außerdem durch den Kerr-Effekt bestimmt werden. Streng genommen, bezieht sich der Kerr-Effekt auf die ,,elektrische“, die Rayleighsche Streuung auf die ,optische" Polarisierbarkeit. Der Unterschied ist aber im allgemeinen sehr gering.
Unter diesen Annahmen ist Tab. 1 aufgestellt worden. Man sieht aus ihr, daß es in der Tat möglich ist, vernünftige Werte der Parameter zu finden, die die Beobachtungen für die in der rechten Spalte angegebenen Größen darstellen ${ }^{10}$. In manchen Fällen sind zwei mögliche Lösungen angegeben worden.

Als Hauptergebnis ist anzusehen, daß die Einführung von $\beta$ oder $\gamma$ und von $\alpha^{\prime} \neq 0$ notwendig ist, um die Intensitäts- und Polarisationsangaben mit einem einheitlichen Modell beschreiben zu können. In den meisten Fällen scheint man mit $\gamma=1$ auszukommen.

Die Moleküle CO und NO fügen sich nur teilweise in den allgemeinen Rahmen, insofern ein offensichtlich zu großer Unterschied zwischen $\alpha_{1}$ und $\alpha_{2}$ erhalten wird. Nur wenn von den experimentellen Daten $A$ und $B$ weggelassen werden, erhält man annehmbare Werte ${ }^{2}$.

Auch für OCS ist noch keine völlig befriedigende Lösung gefunden, die alle Beobachtungen $\left(\mu ; \mu^{\prime}\right.$ und $A^{\prime} \mid B^{\prime}$ für zwei verschiedene Schwingungen) genau wiedergibt. Immerhin ist die Diskrepanz der aus den Ultrarot- und den Raman-Beobachtungen errechneten Parameter nicht sehr groß ${ }^{2}$. Völlig gesichert ist das Ergebnis, daß eine der beiden totalsymmetrischen Schwingungen, gegen die übliche Erwartung, einen relativ hohen Depolarisationsgrad im Raman-Effekt besitzen kann, wie er tatsächlich beobachtet wurde.

Einige der Diskrepanzen können auch dadurch bedingt sein, daß die Beobachtungen sich auf ganz verschiedene Spektralgebiete beziehen, oder daß einige experimentelle Werte unsicherer sind, als gewöhnlich angenommen wird, insbesondere $\varrho_{\text {Rayleigh }}$.

Noch eine Bemerkung allgemeiner Art: Das hier behandelte Modell ist ein klassisches. Es umfaßt weder quantenmechanische Resonanzeffekte, noch ist es als Modell solcher Effekte gedacht. Deren Existenz ist ja auf ganz unabhängige Weise nachgewiesen. Immerhin zeigt das Modell, daß Dipolmomente kleiner als $e / r$ nicht von sich aus schon ausreichen, den Grad der Mitwirkung von Resonanz quantitativ abzuschätzen.

Im folgenden sollen zwei spezielle Anwendungen qualitativer Art besprochen werden, die sich beide auf die Gln. (la) und (2a) beziehen.

10 Für die experimentellen Daten vergleiche man die in Tab. 1 und in den Arbeiten der Anm. 2 angegebene Literatur. 


\begin{tabular}{|c|c|c|c|c|c|c|c|c|c|c|}
\hline Molekül & \multicolumn{3}{|c|}{$\alpha\left(10^{-24} \mathrm{~cm}^{3}\right)$} & \multicolumn{3}{|c|}{$\alpha^{\prime}\left(10^{-16} \mathrm{~cm}^{2}\right)$} & $\beta$ & $\gamma$ & $\sigma$ & $\begin{array}{c}\text { Verfügbare } \\
\text { experimentelle } \\
\text { Bestimmungsstücke }\end{array}$ \\
\hline \multirow[t]{2}{*}{$\begin{array}{l}\mathrm{H}_{2} \\
\mathrm{~N}_{2} \\
\mathrm{O}_{2} \\
\mathrm{Cl}_{2}\end{array}$} & \multicolumn{3}{|c|}{$\begin{array}{l}0,4 \\
0,41 \\
0,83 \\
0,96 \\
0,72 \\
0,92 \\
2,1 \\
2,3\end{array}$} & \multicolumn{3}{|l|}{$\begin{array}{l}0,71 \\
1,23 \\
0,66 \\
0,49\end{array}$} & $\begin{array}{l}0,085 \\
0,07 \\
0,24 \\
0,32 \\
0,49 \\
0,24 \\
0,65 \\
0,48\end{array}$ & $\begin{array}{l}0,085 \\
0,14 \\
0,24 \\
0,64 \\
0,49 \\
1,0 \\
0,65 \\
1,0\end{array}$ & $\begin{array}{l}- \\
- \\
- \\
- \\
-\end{array}$ & $\begin{array}{l}A, B \\
A, B, A^{\prime} / B^{\prime} \\
A, B, A^{\prime} / B^{\prime} \\
A, B\end{array}$ \\
\hline & & $\alpha_{0}$ & $\alpha_{\mathrm{s}}$ & & $\alpha^{\prime}$ & $\alpha_{\mathrm{S}}^{\prime}$ & & & & \\
\hline \multirow[t]{2}{*}{$\begin{array}{l}\mathrm{CO}_{2} \\
\mathrm{CS}_{2}\end{array}$} & $\begin{array}{l}1,0 \\
1,0\end{array}$ & $\begin{array}{r}1,0 \\
-\end{array}$ & 2,3 & $\begin{array}{l}1,3 \\
0,6\end{array}$ & $\begin{array}{r}3,0 \\
-\end{array}$ & - & $\begin{array}{l}0,10 \\
0,27\end{array}$ & $\begin{array}{l}1,0 \\
1,0\end{array}$ & 0,70 & $\begin{array}{l}A, B, A^{\prime} \mid B^{\prime}, \mu^{\prime} \\
\mu_{\mathrm{Co}}=0,6 \mathrm{D}^{*} \\
A, B, A^{\prime} \mid B^{\prime}, \mu^{\prime} \\
\mu_{\mathrm{Cs}}=0,6 \mathrm{D}^{*}\end{array}$ \\
\hline & $x_{\mathrm{H}}$ & $\alpha_{\mathrm{Hal}}$ & & \multicolumn{3}{|c|}{$\alpha_{1}{ }^{\prime}+\alpha_{2}{ }^{\prime}$} & & & & \\
\hline $\begin{array}{l}\mathrm{HBr} \\
\text { HJ }\end{array}$ & $\begin{array}{l}0,4 \\
0,4\end{array}$ & $\begin{array}{l}2,72 \\
4,23\end{array}$ & & \multicolumn{3}{|c|}{ 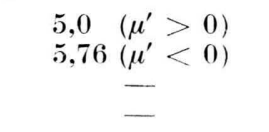 } & $\begin{array}{l}0,42 \\
0,44 \\
0,63\end{array}$ & $\begin{array}{l}1,0 \\
1,0 \\
1,0\end{array}$ & $\begin{array}{l}0,8 \\
0,8 \\
0,89\end{array}$ & $\begin{array}{l}A, B^{* *}, \mu \\
\mu^{\prime}= \pm 0,95 \mathrm{D} / \AA * * * \\
A, B, \mu \\
A, B, \mu\end{array}$ \\
\hline
\end{tabular}

* D Z. Robinson, Review Papers from a Symposium on Molecular Structure, Ohio State Univ., June 1951; herausgeg. vom Office of Naval Research, Washington, D. C.

** Landolt-Börnstein, Physik.-chem. Tab. 6. Aufl., Band I 3, S. 510 ff., Springer, Berlin 1951.

*** H. Rollefson. Symposium on Molecular Structure, vorgetragen am 12. Juni 1951 .

Tab. 1. Polarisierbarkeiten und Abschirmfaktoren.

\section{Anwendungen}

\section{a) Homöopolare Moleküle}

Es ist bekannt, daß auch homöopolare Stoffe wie $\mathrm{FCl}$ oder $\mathrm{JCl}$, eine merkliche Ultrarotabsorption besitzen. Besonders auffällig ist ferner das Beispiel von $\mathrm{SiC}$ (Carborund), das eines der stärksten Reflexionsmaxima aufweist, die je im Ultrarot beobachtet wurden. Diese Ultrarot-Aktivität soll hier interpretiert werden als herrührend von induzierten Dipolen, obwohl man geneigt ist, solcher Strahlung nur einen Effekt zweiter Ordnung zuzuschreiben. Zwar sind FCl und JCl erfahrungsgemäß Dipolmoleküle ${ }^{11}$. Man muß dann aber die Frage stellen, woher das Dipolmoment kommt, das zu der Ultrarotabsorption Anlaß gibt.

In unserem Modell gibt Gl. (1 a) die Antwort. Wenn man nun für $\alpha$ und $\alpha^{\prime}$ Werte derselben Größenordnung einsetzt wie in der Tab. 1 , so erhält man $\mu^{\prime}$ ebenfalls in derselben Größenordnung, wie sie an anderen, „,normalen“ Dipolgasen beobachtet wurden. Da quantitative Angaben über $\mu^{\prime}$ fehlen, können keine genaueren Aussagen gemacht werden.

\footnotetext{
11 Homöopolare Stoffe, in unserer Bezeichnungsweise, sind also nicht notwendigerweise unpolar.
}

Ähnliche Verhältnisse sind auch für $\mathrm{SiC}$ zu erwarten, wo aber die Kristallstruktur die Dinge kompliziert.

Nur wenn die beiden Atome identisch sind (gleiches $\alpha$ und gleiches $\alpha^{\prime}$ ), ist die induzierte Dipolstrahlung allgemein vernachlässigbar, wenigstens unter normalen Bedingungen. In allen anderen Fällen kann sie mit normaler Dipolstrahlung vergleichbar sein. Im übrigen folgt aus (1) und (2), bzw. (1 a) und (2a), daß die Intensität einer Schwingungsbande nicht mit dem Dipolmoment parallel gehen muß.

Man könnte annehmen, daß auch die individuellen atomaren Dipole eine ähnliche Strahlung emittieren oder absorbieren. Nun sind aber die individuellen Dipole im Molekül oder im Kristall nicht unabhängig voneinander, und in großen Abständen vom Molekül wird ihre Strahlung durch Interferenz vernichtet. Daher strahlt nur der molekulare Dipol als Ganzes. Anders ausgedrückt, man beobachtet nur die Differenz der Strahlung der atomaren Dipole, was ja durch die Form von Gl. (2a) angedeutet ist.

Tatsächlich beobachtet man schwache selektive Absorption auch in Gasen wie $\mathrm{O}_{2}$ oder in Kristallen 
wie Diamant oder Silicium. Dort hat man es entweder mit Quadrupolstrahlung zu tun oder, bei hohen Dichten, mit induzierter Dipolstrahlung, die infolge Störung der Symmetrie durch Nachbarmoleküle bzw. durch Kristallbaufehler beobachtbar wird. Es ist allerdings bemerkenswert, da $ß$ absichtliche Vermehrung von Strukturfehlern anscheinend ohne wesentlichen Einfluß auf das Spektrum ist ${ }^{12}$. Möglicherweise sind daher die immer vorhandenen Strukturschwankungen infolge der niederfrequenten ,,akustischen" Schwingungen verantwortlich zu machen. Wegen der Anharmonizität der Bindung sind diese Schwankungen. der Symmetrie wahrscheinlich nicht ohne Einfluß auf die Intensitäten.

\section{b) Disubstituierte Benzole}

Die Absorption disubstituierter Benzole $\mathrm{X} \cdot \mathrm{C}_{6} \mathrm{H}_{4} \cdot \mathrm{Y}$ bei etwa $6,2, \mu$ zeigt einige charakteristische Eigenschaften, die offensichtlich eng mit der Art der Substituenten verknüpft sind ${ }^{13}$. Das Intensitätsverhältnis $I_{o}: I_{m}: I_{p}$ für ortho-, meta- und para-Substitution ist einerseits abhängig von den individuellen Substituenten. Diese individuellen Eigenschaften sollenhier nicht betrachtet werden. Anderseits lassen sich die Intensitätsverhältnisse gruppenweise zusammenfassen, je nach dem Typus der Substituenten in bezug auf „Substitutions-Direktion“. Man unterscheidet ,,metadirigierende" und ,ortho-para-dirigierende" Substituenten, je nachdem der zweite Substituent bevorzugt in der meta- oder in der ortho- bzw. paraStellung substituiert wird. Die Dirigierfähigkeit kann mehr oder weniger stark sein. Wir erhalten demnach folgende Gruppen von Di-Derivaten: $m-m ; o, p-o, p ; m-o, p ; \sim-m ; \sim-o, p$; $\sim \sim \sim$; in leicht verständlicher Bezeichnung, wenn $\sim$ einen Substituenten von schwacher Dirigierfähigkeit andeutet. Beobachtungen liegen vor für die Gruppen $m-m$ (1 Beispiel), $o, p-o, p$ (10

\begin{tabular}{|c|c|c|c|}
\hline Gruppe & $\begin{array}{c}o, p-o, p \\
m-m\end{array}$ & $m-o, \nu$ & $\cdots-m$ \\
\hline$I_{n}: I_{m}: I_{p}$ & $\begin{array}{c}a: b: a^{*} \\
b \geqslant a\end{array}$ & $\begin{array}{c}a: b: a \\
b-a\end{array}$ & $\begin{array}{c}a: a: b \\
b=2 a\end{array}$ \\
\hline
\end{tabular}

* Wesentliche Ausnahmen: $\mathrm{mit}_{3} \cdot \mathrm{C}_{6} \mathrm{H}_{4} \cdot \mathrm{NH}_{2}$ und $\mathrm{NO}_{2} \cdot \mathrm{C}_{6} \mathrm{H}_{4} \cdot \mathrm{NO}$.

Tab. 2. Intensitätsverhältnisse in disubstituierten Benzolen bei $6,2 \mu$.

12 Ich danke Herrn Dr. J. R. Collins, Purdue Univ., Lafayette, Indiana, für freundliche Mitteilung dieser Beobachtung.

${ }^{13}$ Herrn Dr. J. Bomstein, Sinclair Research Lab.,
Beispiele), $m-o, p$ (3 Beispiele) und - $m$ (2 Beispiele). Das qualitative Resultat zeigt Tab. 2 in stark schematisierter Form.

Zur Deutung dieser Verhältnisse müssen herangezogen werden:

1. Die Schwingungsform, die in Abb. 1 für eine ,Ringschwingung" des Benzolgerüsts mit den für die $6,2 \mu$-Bande wahrscheinlich zutreffenden Symmetrieeigenschaften angedeutet ist. Die Abb. 1 gibt nur die allgemeinen Symmetrieverhältnisse dieser Schwingung an. Die einzelnen Amplituden hängen von den Substituenten ab, ohne daß genauere Angaben möglich wären. Schon aus diesem Grunde verbietet sich eine individuelle und quantitative Behandlung.

Abb. 1. Schematische Schwingungsform der 6,2- $\mu$-Bande des Benzolrings.

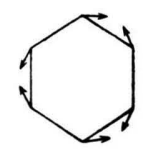

2. Die Polarisierbarkeiten der C-Atome im Ring. Diese können als verschieden angesehen werden je nach den verschiedenen Substituenten. Wir unterscheiden $\alpha_{X}, \alpha_{Y}, \alpha_{0}$ für X-Substitution, Y-Substitution und Nicht-Substitution. Wir vernachlässigen alle Wechselwirkung, obwohl, insbesondere bei ortho-Derivaten, eine solche nicht grundsätzlich auszuschließen ist.

3. Der Typus der Substituenten. Dieser beeinflußt die Ladungsverteilung im Ring ${ }^{14}$. In starker Vereinfachung der Dinge kann gesagt werden, daß ein $o, p$-dirigierender Substituent den C-Atomen, die in ortho- oder para-Stellung zu ihm sind, eine positive Zusatzladung gibt. Ein meta-dirigierender Substituent ruft an denselben Atomen negative Ladungen hervor. Von einem schwach dirigierenden Substituenten wollen wir vereinfachend annehmen, daß er die normale Ladungsverteilung überhaupt nicht beeinflußt.

Wir behandeln die Schwingung als eine reine CSchwingung, bei der der Einfluß der Substituenten allein durch die Verschiedenheit der $\alpha$-Werte und durch die Erzeugung von relativen Zusatzladungen in Rechnung gesetzt wird.

Wenn wir $\mu$ und $\mu^{\prime}$ für den Benzolring nach den oben beschriebenen Methoden berechnen [als Vektorsumme von Bindungsmomenten auf Grund der

Harvey, Illinois, danke ich herzlich für die Mitteilung seiner Ergebnisse.

${ }^{14}$ Vgl. z. B. L. Pa uling, The Nature of the Chemical Bond, Cornell University Press, Ithaca 1945, p. $149 \mathrm{ff}$. 
Gl. (1 a) und (2a)], zunächst ohne Berücksichtigung der Zusatzladungen, so erhalten wir mit

$\xi=\frac{\alpha_{\mathrm{x}}{ }^{\prime}-\alpha_{\mathrm{o}}{ }^{\prime}}{r^{2}}-\frac{2\left(\alpha_{\mathrm{x}}-\alpha_{\mathrm{c}}\right)}{r^{3}}$.

$$
\eta=\frac{\alpha_{\mathrm{r}}{ }^{\prime}--\alpha_{0}{ }^{\prime}}{r^{2}}-\frac{2\left(\alpha_{\mathrm{r}}-a_{0}\right)}{r^{3}} .
$$

für ortho-Derivate.

$$
\mu_{0}^{\prime} \text { ।I }=2 e(\xi-\eta), \mu_{0}^{\prime}=\frac{\mid \overline{3}}{2} e(\xi+\eta) .
$$

parallel und senkrecht zur Achse $3-6$ in üblicher Numerierung;

für meta-Derivate,

$$
u_{m \| 1}^{\prime}=\frac{1}{2} e(\zeta-\eta), \mu_{m \perp}^{\prime}=\frac{\sqrt{3}}{2} e(\xi+\eta) .
$$

parallel und senkrecht zur Achse 2 - 5:

für para-Derivate,

$$
\mu_{p}^{\prime}=\sqrt{3} e(\xi+\eta),
$$

senkrecht zur Achse $1-4$.

Der Einfachheit halber sind alle Amplituden als gleich angenommen worden und alle etwaigen $\mathrm{Ab}$ schirmfaktoren gleich 1 gesetzt worden.

Wenn $\xi=\eta$, d. h. für $\mathrm{X}=\mathrm{Y}$, erhalten wir

$$
\mu_{o}^{\prime}: \mu_{m}^{\prime}: \mu_{p}^{\prime}=1: 1: 2,
$$

also genau das für die Gruppe - $-m$ in Tab. 2 angegebene Resultat. Die Übereinstimmung der Zahlenwerte ist Zufall, einerseits wegen der qualitativen Natur der Tab. 2 und anderseits weil $a$ und $b$ in Tab. 2 nicht $\mu^{\prime}$-Werte darstellen, sondern sich auf $\log I_{0} / I$ beziehen. Ferner sind Abweichungen zu erwarten für $\mathrm{X} \neq \mathrm{Y}$ und bei Berücksichtigung der Amplitudenunterschiede. Aber wir wollen annehmen, daß die Berücksichtigung dieser Umstände das Resultat nicht merklich ändern wird.

Aus Abb. 2 folgt nun, daß für die Gruppe $\sim-o, p$ und $\sim-m$ die Zusatzladungen in allen drei Derivaten den gleichen Effekt hervorrufen. Sie können nämlich zusätzliche induzierte Dipole erzeugen, die sich aber im resultierenden Moment nicht bemerkbar machen. Auch für $\mu^{\prime}$ kommen sie in erster Näherung nicht in Betracht. Damit ist für die $(\sim-m)$-Gruppe eine Deutung der Beobachtung gewonnen.

Ein Blick auf Abb. 2 lehrt nun. daß bei den beiden anderen Substitutionsgruppen die Zusatzladungen wesentlich mitwirken, und zwar nimmt in beiden Gruppen das meta-Derivat eine Sonderstellung ein. In der $(o, p-o . p)$-Gruppe wird die Wirkung der Ladungen in den ortho- und para-Derivaten geschwächt (wegen benachbarter gleichnamiger Ladungen), im meta-Derivat verstärkt. Dasselbe gilt für die Gruppe $m-m$. Es ist also zu erwarten, daß das meta-Derivat die größte Intensität zeigt, wenn auch im einzelnen über die Überlagerung des Ladungseffekts mit dem auch hier anzunehmenden Induktionseffekt der $(\sim-m)$-Gruppe nichts ausgesagt werden kann.
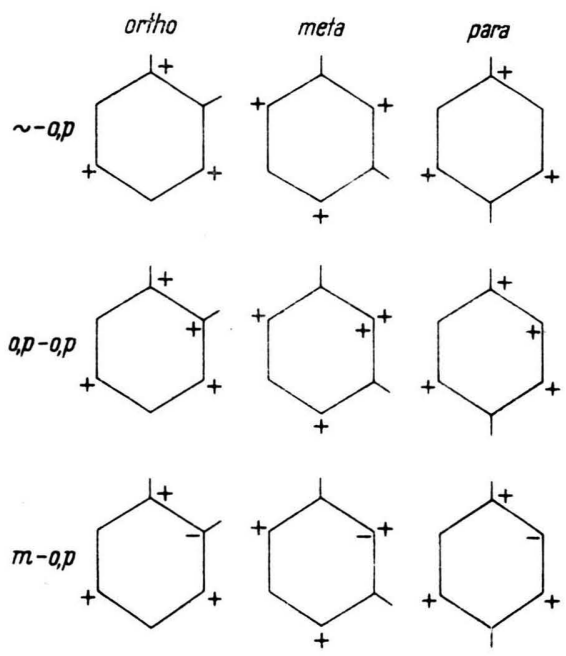

Abb. 2. Schematische Ladungsverteilung in disubstituierten Benzolen. Die vom einen Substituenten erzeugten Ladungen sind im Innern des Rings, die des andern im Äußern angezeigt. Die drei ,,äußeren" Ladungen sind auf verschiedene Moleküle verteilt $\mathrm{zu}$ denken. Für die ,,inneren" Ladungen ist nur eine der 3 möglichen Stellungen gezeichnet. Die anderen Möglichkeiten geben keinen wesentlichen Unterschied. Für die Gruppen $m-m$ und $-m$ ist $\perp$ durch $-7 u$ ersetzen.

Für die $(m-o, p)$-Gruppe ist die Wirkung der Zusatzladungen im meta-Derivat geschwächt ( $\mathrm{Zu}$ sammenfallen ungleichnamiger Ladungen), in den beiden anderen relativ stark durch das Auftreten von Dipolen. In derselben Weise interpretiert wie in dem vorhergehenden Absatz, bedeutet dies ein der Beobachtung völlig entsprechendes Intensitätsverhältnis.

Damit ist ein qualitatives Verständnis der charakteristischen Unterschiede der Intensitätsverteilung in den verschiedenen disubstituierten Benzolen erzielt, soweit es den allgemeinen Typus betrifft. Angesichts der starken Vereinfachungen des Problems ist es jedoch verfrüht. auch die individuellen Eigenheiten zu erfassen. 
Zusatz b. d. Korr.: L. Couture-Mathieu und J.-P. Mathieu (Acta Cryst. 5, 571 [1952]) haben kürzlich Werte für die Komponenten des Tensors der Änderung der Polarisierbarkeit für das $\mathrm{H}_{2} \mathrm{O}$-Molekül in $\mathrm{LiClO}_{4} \cdot 3 \mathrm{H}_{2} \mathrm{O}$ angegeben, die sich gut in das hier betrachtete Modell einfügen. Sie erhalten für die symmetrische Valenzschwingung $\varepsilon_{x x}: \varepsilon_{y y}: \varepsilon_{z z}=0,26: 0,19$ : 0,78 oder $0,78: 0,19: 0,26$. Das Modell liefert aus $\varepsilon_{x x} / \varepsilon_{z z}=1 / 3 \quad\left(\right.$ mit $\left.\quad \alpha_{\mathrm{H}}=0,4, \quad \alpha_{\circ}=0,75, \quad \beta=\gamma=1\right)$
$\alpha_{\mathrm{H}}^{\prime}=1,68, \alpha_{\mathrm{o}}^{\prime}=1,64$. Hieraus folgt $\varepsilon_{y y}=0,28$. Die Alternative $\varepsilon_{x x} / \varepsilon_{z z}=3$ gibt keine mögliche Lösung. Die Diskrepanz für $\varepsilon_{y u}$ kann wohl durch geeignete Wahl von $\beta$ verringert werden, jedoch lohnen sich die umständlichen Rechnungen zur Zeit nicht. Die Angabe von Couture und Mathieu, da $\beta \varepsilon_{x x}$ und $\varepsilon_{y y}$ entgegengesetzte Vorzeichen haben, beruht auf einem Versehen. Aber selbst dann wäre eine Darstellung im Rahmen des Modells möglich.

\title{
Entropie der Elektronen in Metallgittern
}

\section{Von Ulrich Dehlinger}

\author{
Aus dem Institut für theoretische und angewandte Physik \\ der Technischen Hochschule, Stuttgart \\ (Z. Naturforschg. 8a, 67-69 [1953]; eingegangen am 1. September 1952)
}

$$
\text { Erwin Fues zum 60. Geburtstag }
$$

\begin{abstract}
Wenn die Fermi-Grenze mit steigender Temperatur schmale Bänder durchschreitet, entsteht eine anomale spezifische Wärme, deren Betrag gegenüber der sonstigen nicht zu vernachlässigen ist. Außerdem erhält das Elektronensystem dann ęine mit der Symmetrie und Koordinationszahl oder mit dem Paramagnetismus des Gitters zusammenhängende Entropie, die die allotropen Umwandlungen, vor allem beim Eisen, hervorrufen kann.
\end{abstract}

$\mathrm{S}_{\mathrm{H}}$ chon vor Jahren hat der Verf. vorgeschlagen, zur Erklärung der Allotropie metallischer Elemente und Verbindungen eine dem Elektronensystem hochsymmetrischer Gitter zukommende Entropie heranzuziehen ${ }^{1}$. Es haben nämlich sämtliche Elemente, die in mehreren Modifikationen kristallisieren, bei höherer Temperatur ein Gitter mit höherer Symmetrie oder mit größerer Koordinationszahl als bei tieferen Temperaturen ${ }^{2}$. Dabei ist diese Art von Allotropie besonders häufig im Bereich der Übergangsmetalle mit ihren nicht abgeschlossenen dSchalen. Da nun die Symmetrie der Atom-Eigenfunktionen nicht abgeschlossener Schalen nicht ohne weiteres ${ }^{3}$ mit der Punktsymmetrie des Atoms in einem hochsymmetrischen Gitter übereinstimmt, wird es vielfach verschiedene Einstellungsmöglichkeiten der Atomfunktionen geben und damit eine Entropie des Elektronensystems auftreten, die in bekannter Weise die thermodynamische Stabilität des betreffenden Zustands bei höherer Temperatur erhöht. Da die Umwandlungswärme der allotropen Modifikationen der Elemente untereinander einige hundert g-cal je Mol beträgt, genügt eine Entropie-

${ }^{1}$ U. Dehlinger, Z. Physik 94, 231 [1935].

2 Vgl. z. B. E. B randenberger, Grundriß der allgem. Metallkde., Reinhardt, München und Basel 1952. Ausnahmen bilden das $\mathrm{Li}$, das bei sehr tiefen Temperaturen ein flächenzentriertes Gitter hat, und das Ca, differenz von der Größenordnung $R \ln 2$, wie sie nach diesen Überlegungen zu erwarten ist, um einen Umwandlungspunkt von mehreren hundert Grad Kelvin $\mathrm{zu}$ erzeugen.

Dieser Versuch, Allotropie bei Metallen nicht auf Gitterschwingungen, sondern auf Änderung der Elektronenzustände zurückzuführen, begegnete u.a. folgendem Einwand: Am absoluten Nullpunkt ist die quantenmechanische Entartung der Elektronenzustände aufgehoben und damit ihre Entropie für jeden tatsächlich dort noch existierenden Zustand sicher gleich Null. Wenn also bei einer höheren Temperatur das Elektronensystem eine Entropie besitzt, so muß zwischen absolutem Nullpunkt und dieser Temperatur eine anomale spezifische Wärme der Elektronen aufgetreten sein, als deren Integral $\int^{T}(c / T$ $\int_{0}^{T}(c / T) \mathrm{d} T$ die Entropie erscheint. Nun ist aber nach der Fermi-Statistik die spezifische Wärme der Metallelektronen so klein, daß auch ihre Entropie nur bei sehr hohen Temperaturen nicht vernachlässigbar kleine Werte annehmen kann.

Ein spezieller Fall dieser Art zeigt aber, daß

das mit steigender Temperatur von der kubischen in die hexagonale dichteste Packung übergeht. Der letztere Fall ist vermutlich mit dem Utbergang eines din ein s-Elektron verknüpft.

${ }^{3}$ Vgl. F. Hund, Z. Physik 99, 119 [1936]. 